\title{
Ambient Moisture Accelerates Hydroamination Reactions of Vinylarenes with Alkali-Metal Amides under Air
}

\author{
Florian F. Mulks, ${ }^{[a]+}$ Leonie J. Bole, ${ }^{[a]+}$, Laia Davin, ${ }^{[c]}$ Alberto Hernán-Gómez, ${ }^{[c]}$ Alan Kennedy, ${ }^{[c]}$ Joaquín \\ García-Álvarez, ${ }^{*[b]}$ and Eva Hevia*[a,c]
}

Dedicated to the memory of Prof Dr. Killian Muñiz

\begin{abstract}
A straightforward alkali-metal-mediated protocol for hydroamination of styrenes using biorenewable 2-methyltetrahydrofuran as a solvent is reported. Refuting the conventional wisdom of the incompatibility of organolithium reagents with air and moisture, we show that the presence of moisture is key in favouring formation of the target phenethylamines over competing olefin polymerisation products. The method is also compatible with sodium amides, with the latter showing excellent promise as highly efficient catalysts under inert atmosphere conditions.
\end{abstract}

The dogma of the need of inert conditions and cold temperatures in reactions with polar main group organometallics has recently been challenged. Addition reactions of Grignard reagents as well as organolithium compounds have been established in the presence of water, in air, and at room temperature. ${ }^{[1]}$ Smooth and expeditious reaction progress was found for addition reactions to ketones, imines, and nitriles. It was discovered that reaction rates with the substrate exceed that found for hydrolysis, leading to high yields of addition products, which were subsequently hydrolysed by the present polar reaction medium. Based on this, deep eutectic solvents (DES) and glycerol have been shown to work even better in these types of reaction due to their lower acidity and lower miscibility with the organic phase. ${ }^{[1 b-e]}$ We have recently been able to perform polymerisation reactions of styrenes on DES in air using RLi reagents as initators with good results. ${ }^{[1 \mathrm{e}]}$

This prompted us to investigate lithium-mediated hydroamination reactions of styrenes in sustainable and aerobic conditions which require the stabilisation of reactive species for prolonged periods of time. While hydroamination reactions have received considerable attention in chemical research for decades, they still remain challenging due to high activation barriers coupled with a flat energy profile, and thus, they continue to attract further investigation. ${ }^{[2]}$ A remarkable number of catalytic protocols have recently been developed using lithium as an alternative to

[a] Dr. F. F. Mulks, Miss L. J. Bole, Prof. Dr. E. Hevia

Departement für Chemie und Biochemie (DCB)

Universität Bern

Freiestrasse 3, 3012 Bern, Switzerland

E-mail: eva.hevia@dcb.unibe.ch

[+] These authors contributed equally to this work.

[b] Dr. J. García-Álvarez

Laboratorio de Compuestos Organometálicos y Catálisis (Unidad

Asociada al CSIC). Departamento de Química Orgánica e

Inorgánica, (IUQOEM), Centro de Innovación en Química

Avanzada (ORFEO-CINQA), Facultad de Química, Universidad

de Oviedo, E-33071, Oviedo, Spain.

E-mail: garciajoaquin@uniovi.es

[c] Dr. L. Davin, Dr. A. Hernán-Gómez, Prof. Dr. Eva Hevia

Department of Pure and Applied Chemistry

University of Strathclyde

295 Cathedral St, G11XL, Glasgow transition metal catalysts. (Scheme 1). ${ }^{[3]}$ Since establishing efficient main group bimetallic catalysts for hydroamination reactions, ${ }^{[4]}$ we have become interested in developing s-block organometallic approaches that are operationally simple for this atom-efficient transformation dedicated to practical everyday laboratory use. Lithium amides are simple-to-synthesize and simple-to-use reactants for such reactions. ${ }^{[5]}$ Herein we report a new method for hydroaminations of styrenes with an exceptional procedural simplicity with cheap and available commodity chemicals using a biorenewable solvent compatible with the presence of air and moisture (Scheme 1). Addition reactions to $\mathrm{C}=\mathrm{C}$ bonds proceed much slower than to their more polar $\mathrm{C}=\mathrm{O}$ and $\mathrm{C}=\mathrm{N}$ counterparts, and hence, controlling the kinetics of the desired reaction versus hydrolysis/degradation of the reactants poses an even greater challenge.

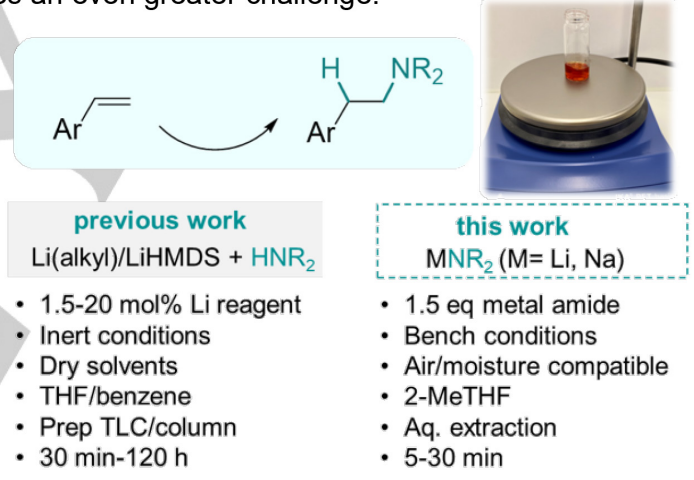

Scheme 1. Comparison of state-of-art catalytic procedures with our air- and moisture compatible stoichiometric protocol.

Phenethylamines are valuable building blocks for general chemical synthesis as well as for pharmaceuticals. ${ }^{[6]}$ Furthermore this study extends the applications of 2-methyltetrahydrofuran, (2-MeTHF), which is produced from biomass degradation products, as a prime solvent for organometallic reactions in air. ${ }^{[7]}$ Recent reports have shown that many polar organometallic reagents are often even more soluble and more stable in 2MeTHF than in THF. ${ }^{[8]}$ We were even able to perform fast amidations of carboxylic acid esters and amides with lithium amides in 2-MeTHF in air. ${ }^{\left[{ }^{[9]}\right.}$ 2-MeTHF is also insoluble in water which conveniently allows more efficient and simple aqueous extraction of reaction crudes without prior solvent exchange.

We started this investigation by dissolving neat $n$-BuLi in 2-MeTHF dried over molecular sieve and added commercial grade amines at $0{ }^{\circ} \mathrm{C}$ drop-wise over 1 min to produce $1.1-1.5 \mathrm{M}$ solutions of the lithium amides $2{ }^{\left[{ }^{10]}\right.}$ Being slightly more electronrich than parent THF, 2-MeTHF exhibited a better solubility of lithium amides and is more suited for the intermediary storage of the generated solutions. The commercially available solvent was stored over molecular sieves to maintain low residual moisture. 
Within only $2 \mathrm{~h}$ an excellent hydroamination yield of $90 \%$ was found when stirring 1.5 eqs. of the $1 \mathrm{M}$ solution of LiPip 2a with styrene 1a under air (Table 1, Entry 1). The conventional solvents THF and $n$-hexane showed reduced performance compared to 2MeTHF. Thus, $79 \%$ yield was found for $3 a$ using THF (Entry 3 and SI for details). Low solubility of amide 2 a led to only $30 \%$ in $n$ hexane (Entry 4, diluted to $0.5 \mathrm{M}$ to counterbalance low solubility). Neither slowing down the reaction by dilution in respect to $\mathbf{2} \mathbf{a}$ from $1 \mathrm{M}$ to $0.375 \mathrm{M}$ nor addition of $1 \mathrm{eq}$. of water significantly impacted the yield, resulting in $82 \%$ (Entry 2 ) and $77 \%$ (Entry 5 ) yields of 3a. This indicates high kinetic stability of $2 \mathbf{a}$ in 2-MeTHF. The presence of $1 \mathrm{~mL}$ of water or ethylene glycol (EG), however, hindered the reaction completely by likely hydrolysing $2 \mathrm{a}$ at a rate faster than the hydroamination reaction (entries 6-7). Despite their slow reaction rate, hydroamination reactions with lithium amide $2 \mathbf{a}$ were compatible with glycerol (Gly) and several DESs, the nonaqueous DESs produced fair to good yields (22-69\%) although sometimes suffering reproducibility issues $(\mathrm{ChCl}$ : choline chloride Entries 9-11). It is noteworthy that stability of the lithium amide in air for such long reaction times is surprising. Interestingly, if the reaction of $\mathbf{1} \mathbf{a}$ with $\mathbf{2} \mathbf{a}$ is carried out in $2-\mathrm{MeTHF}$ in air but placing a drying tube charged with $\mathrm{CaCl}_{2}$ in top of the reaction vial, the yield of $3 a$ diminishes from $90 \%$ to a modest $35 \%$ (Entry 12), which hints at an unexpected beneficial effect of moisture in these reactions.

Table 1. Influence of the reaction time on the yield of the addition of LiPip 2a to 4-methylstyrene $1 \mathrm{a}$.

\begin{tabular}{|c|c|c|c|}
\hline Entry & Medium A & Medium B & Yield [\%] \\
\hline 1 & $1.5 \mathrm{~mL}$ 2-MeTHF & - & 90 \\
\hline 2 & $4.0 \mathrm{~mL}$ 2-MeTHF & - & 82 \\
\hline 3 & $1.5 \mathrm{~mL}$ THF & - & 79 \\
\hline 4 & $3.0 \mathrm{~mL} n$-hexane & - & $19-32^{[a, b]}$ \\
\hline 5 & $1.5 \mathrm{~mL}$ 2-MeTHF & 1 eq. $\mathrm{H}_{2} \mathrm{O}(18 \mu \mathrm{L})$ & 77 \\
\hline 6 & $1.5 \mathrm{~mL}$ 2-MeTHF & $1 \mathrm{~mL} \mathrm{H} \mathrm{H}_{2} \mathrm{O}$ & 0 \\
\hline 7 & $1.5 \mathrm{~mL}$ 2-MeTHF & $1 \mathrm{~mL}$ EG & 2 \\
\hline 8 & $1.5 \mathrm{~mL}$ 2-MeTHF & $1 \mathrm{~mL}$ Gly & $44-69^{[a, b]}$ \\
\hline 9 & $1.5 \mathrm{~mL}$ 2-МеTHF & $1 \mathrm{~mL} \mathrm{ChCl/2Gly}$ & $64^{[b]}$ \\
\hline 10 & $1.5 \mathrm{~mL}$ 2-MeTHF & $1 \mathrm{~mL} \mathrm{ChCl/2EG}$ & $32-46^{[a, b]}$ \\
\hline 11 & $1.5 \mathrm{~mL}$ 2-MeTHF & $1 \mathrm{~mL} \mathrm{ChCl} / 2 \mathrm{H}_{2} \mathrm{O}$ & $22^{[b]}$ \\
\hline 12 & $1.5 \mathrm{~mL}$ 2-MeTHF & & $35^{\text {c] }}$ \\
\hline
\end{tabular}

Reactions performed on a $1 \mathrm{mmol}$ scale stirring at $900 \mathrm{rpm}$. Mean yields of isolated aliquots of at least two independent reactions are given. ${ }^{[a]} \mathrm{A}$ range is given due to the spread of results. ${ }^{[b]}$ Stirred at $500 \mathrm{rpm} .{ }^{[c]}$ Carried out with a drying tube packed with pre-dried $\mathrm{CaCl}_{2}$ attached to the top of the reaction vial.

With the air-and moisture-compatibility of this approach being the most striking difference to other known methods, we also noted a marked effect on the stirring speed of the reaction (Table S1, SI). Thus while vigorous stirring (900 rpm) lead to the formation of $3 \mathbf{a}$ in an $86 \%$ yield after just 10 minutes, if the speed is reduced to $500 \mathrm{rpm}$ the yield observed decreases to $48 \%$ and if stirring is not employed $2 \mathrm{~h}$ are required to achieve a $94 \%$ conversion. Since both lithium amide and styrene are highly soluble in 2-MeTHF, a possible explanation for the accelerating effect observed with the vigorous stirring could be that this facilitates the mixing with ambient moisture. Interestingly, it should be noted that the short reaction times observed for the formation of $\mathbf{3 a}$ while operating at room temperature push this approach into the faster regimes of known methods for lithium-mediated hydroamination of styrenes. ${ }^{[3]}$

Previous work on s-block metal catalysed hydroamination of styrenes have proposed that reaction occurs via initial amine deprotonation by the metal pre-catalyst forming a reactive metal amide, with a highly polarised $\mathrm{M}-\mathrm{N}$ bond which in turn can insert into the $\mathrm{C}=\mathrm{C}$ bond of the substrate furnishing the $\beta$-metallated phenethylamine intermediate I. $^{[11]}$ Protonation of $\mathbf{I}$ by excess amine, regenerates the metal amide and liberates hydroamination product (Scheme 2). Traces of water could quench the lithioaminated species I from the equilibrium, which has been discussed to be only slightly stabilised over the reactants themselves ${ }^{[12]}$ In addition, traces of water could also react with some lithium amide LiPip 2a (which, in our study, is present in a 0.5 eq. excess) to generate free piperidine that could subsequently favour the protonation of $I$. In this regard it should be noted that mechanistic and kinetic studies in group II metal catalysed hydroaminations of alkenes and isocyanates have suggested that these processes take place preferentially via an amine-assisted concerted transition state. ${ }^{[13]}$

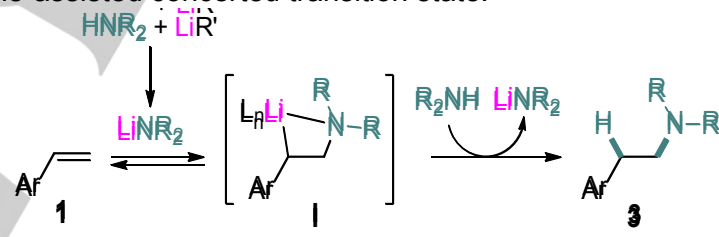

Scheme 2. Proposed mechanism of catalytic hydroamination of styrenes with lithium amides $(L=$ amine or solvent molecule $) .{ }^{[13,14]}$

Further support for the beneficial effect of moisture in these reactions, was found when $1 \mathrm{a}$ and $\mathbf{2 a}$ were reacted under strict inert atmosphere conditions using dried and degassed 2-MeTHF (30 min, $900 \mathrm{rpm}, 1.5$ eqs. of $2 \mathbf{a}$ ), which furnished amine $\mathbf{3 a}$ in a modest $28 \%$ yield (Table 2 , Entry 1 ).

Table 2. Results from addition of LiPip 2a to 4-methylstyrene 1a under dry, wet and excess amine conditions

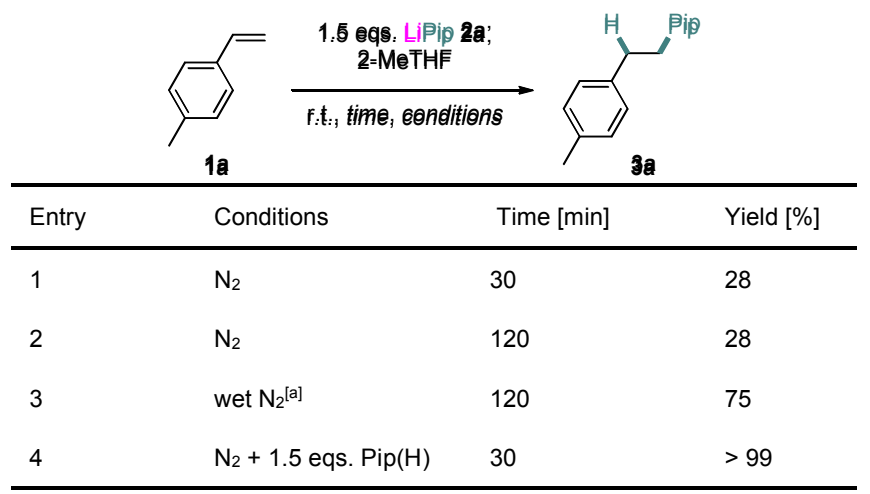

Reactions performed on a $1 \mathrm{mmol}$ scale in a total volume of $1.5 \mathrm{~mL}$ of 2 MeTHF. Mean yields of isolated aliquots of at least two independent 
reactions are given. ${ }^{[a]} \mathrm{A}$ slow flow of $\mathrm{N}_{2}$ over degassed water was run over the reaction mixture.

The same conversion was observed allowing longer reaction times of $2 \mathrm{~h}$. These low yields are in stark contrast with those observed while working under air and in the presence of moisture, conditions typically strictly avoided in organolithium chemistry, which furnished $\mathbf{3 a}$ almost quantitatively. Interestingly if the reaction is carried out under a nitrogen atmosphere but introducing small amounts of water by running a nitrogen flow over degassed water the yield increases from 28 to $75 \%$, supporting the idea that high conversions observed when working in air are related to the moisture present in the reaction media rather than oxygen. When employing a solution of LiPip 2a with a stoichiometric excess of piperidine under inert atmosphere conditions, using dried and degassed 2-MeTHF, we observed $>99 \%$ yield after $30 \mathrm{~min}$ (Table 2, entry 4 ), which is consistent with the notion that excess piperidine accelerates the reaction and that under the conditions of our study, it can be generated by partial hydrolysis of the employed lithium amide.

${ }^{1} \mathrm{H}$ NMR reaction monitoring studies in $\mathrm{D}_{8}$-THF on a $1: 1$ mixture of LiPip 2a and Pip $(\mathrm{H})$ support the initial formation of a co-complex (Figure 1). ${ }^{[14]}$ Thus, as shown in Figure 1ii, a single set of signals is observed for the Pip rings exhibiting a modest but noticeable change on their chemical shifts with respect of those of $\mathbf{2 a}$ (Figure1i). Addition of 0.67 eq of $1 \mathrm{a}$ to this mixture (replicating the conditions of Entry 4 in Table 4) led to the quantitative formation of hydroamination product $\mathbf{3 a}$ along with the recovery of the LiPip.Pip(H) complex (Figure 1iii). We also noted that while under inert atmosphere conditions in $\mathrm{D}_{8}$-THF, $3 \mathbf{a}$ can also be formed by reacting $1 \mathbf{a}$ and piperidine using catalytic amounts of $2 \mathbf{a}(20$ mol\%,> 99\% yield, 10 min, see Fig S116 in SI), in air using 2MeTHF as solvent, 3a was obtained only in a $16 \%$ yield. These findings indicate that our approach only works stoichiometrically, probably due to the competing degradation of LiPip under the conditions employed.

NMR monitoring studies also showed that addition of $1 \mathrm{a}$ to a solution of $2 a$ in $\mathrm{D}_{8}$-THF (or 2-MeTHF) in the absence of amine, under inert atmosphere conditions, produces extensive polymerisation of the styrene. ${ }^{[15]}$
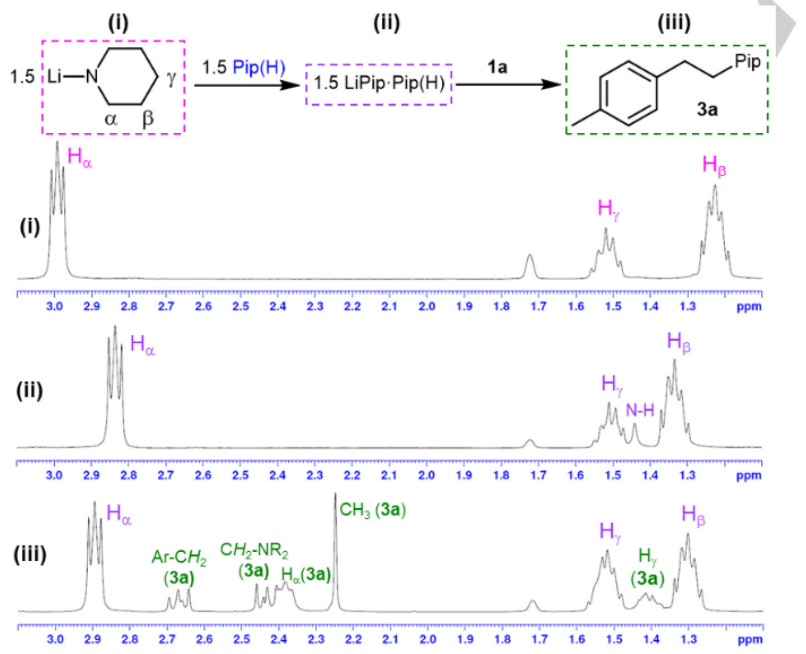

Figure 1. Aliphatic region from ${ }^{1} \mathrm{H}$ NMR spectra $(400 \mathrm{MHz})$ in $\mathrm{D}_{8}$-THF (i) LiPip (2a); (ii) LiPip $2 \mathbf{a}+1$ eq of Pip(H); (iii) LiPip $2 \mathbf{a}+1$ eq of $P i p(H)+0.67$ eq $\mathbf{1 a}$.
In addition, ${ }^{1} \mathrm{H}$ DOSY NMR studies of $\mathbf{2}$ a suggest that in $\mathrm{D}_{8}$-THF solutions this lithium amide exists as a $\left[\left(\mathrm{D}_{8}-\mathrm{THF}\right)_{3} \mathrm{Li}(\mathrm{Pip})\right]$ monomer $\left(M W_{\text {det }}=275 \mathrm{~g} / \mathrm{mol}\right.$, error $-12 \%$, see SI for details $) .{ }^{[16]}$ Formation of small kinetically activated aggregates of $\mathbf{2} \mathbf{a}$ in these ethereal solvents should contribute to the fast reactivity observed in these hydroamination processes. ${ }^{[9]}$ Thus, we propose that when the reaction is carried out under air, the presence of moisture can generate some $\mathrm{Pip}(\mathrm{H})$ that as discussed above coordinates to $\mathbf{2 a}$, which in turn reacts with styrene $\mathbf{1 a}$ to form selectively hydroamination product $3 \mathbf{a}$ via intermediate $\mathbf{I}$. This can be subsequently quenched by $\mathrm{PipH}$ or traces of moisture (Scheme 3). On the contrary, under strict inert atmosphere conditions, using dry solvents, I reacts with free styrene (present in solution due to the equilibrium shown in Scheme 2) initiating its polymerisation. ${ }^{[1 \mathrm{e}, 15]}$

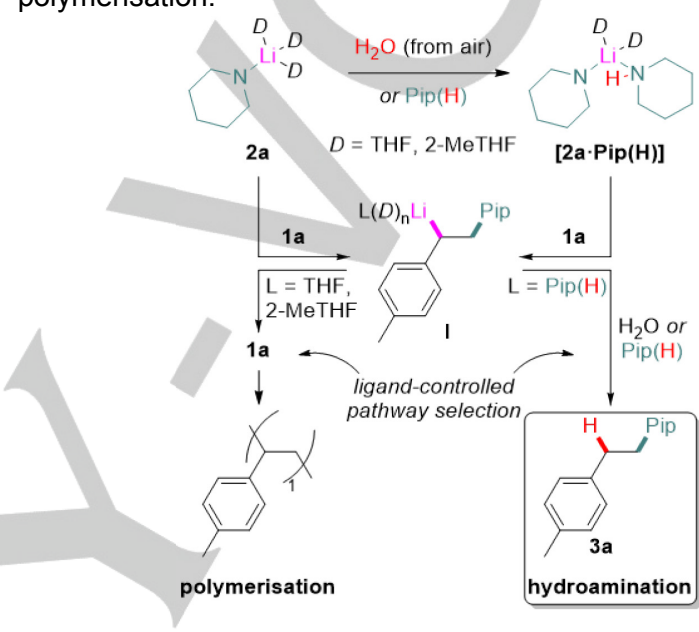

Scheme 3. Reaction showing excess piperidine or ambient moisture supply is necessary to favour hydroamination over polymerisation

We next explored the scope of this air/moisture compatible method towards different amides (Table 3).

Table 3. Reaction scope using different lithium amides 2 with 4-methylstyrene.

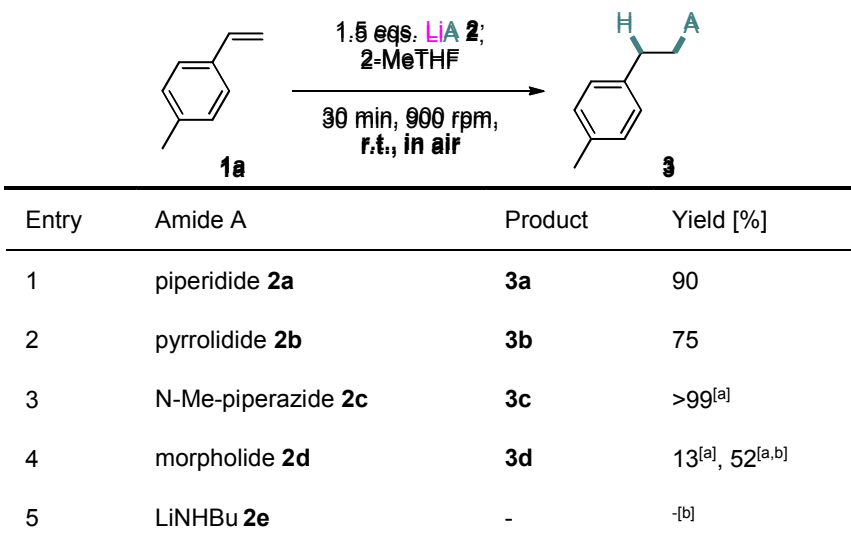

Reactions performed on a $1 \mathrm{mmol}$ scale in $1.5 \mathrm{~mL}$ of 2-MeTHF. They are stirred at $900 \mathrm{rpm}$ for $30 \mathrm{~min}$. Mean yields of isolated aliquots of at least two independent reactions are given. [a] $9 \mathrm{~mL} 2-\mathrm{MeTHF}$ used due to low solubility. ${ }^{[b]} 500 \mathrm{rpm}, 2 \mathrm{~h}$.

At $900 \mathrm{rpm}$ stirring speed and with 30 min of reaction time, we found LiPip 2a gave $90 \%$ yield of the desired terminal amine $\mathbf{3 a}$. The 5 -ring amide lithium pyrrolidide $\mathbf{2} \mathbf{b}$ gave $75 \%$ of $\mathbf{3 b}$. The 
heteroatom substituted lithium $N$-methylpiperazide 2c and lithium morpholide $\mathbf{2 d}$ were poorly soluble in 2-MeTHF. We thus diluted them by a factor of 6 to give more uniform $0.17 \mathrm{M}$ dispersions. A virtually quantitative yield for $3 c$ was found employing this dispersion. On adding morpholide the reaction proceeded slower and gave only $13 \%$ yield of $\mathbf{3 d}$ under our standard conditions, which rose to $52 \%$ by stirring the reaction for $2 \mathrm{~h}$ at a reduced speed of $500 \mathrm{rpm}$ to slow down the moisture intake. The aliphatic amide lithium $n$-butylamide $2 \mathrm{e}$ did not form significant amounts of product under the original conditions nor under reduced stirring speed $(500 \mathrm{rpm})$ and longer reaction time $(2 \mathrm{~h})$. Broad signals in the ${ }^{1} \mathrm{H}$ NMR spectra of the reaction mixtures suggest that this amide might oligomerise the styrene before the action of ambient moisture generated enough of the corresponding amine. We also assessed the reactivity of $\mathbf{2 a}$ towards several styrenes (Table 4) Steric repulsion, unsurprisingly, leads to significant yield reductions. With these reactions being marginally energetically downhill, steric bulk leads to incomplete conversion of the substrates. While we found an excellent yield of $90 \%$ for unsubstituted styrene 1c, ortho-methyl styrene $1 \mathrm{~d}$ gave $54 \%$ and 1,1-diphenyl styrene $1 \mathrm{~b}$ yielded $41 \%$. Other vinylic methyl and phenyl substituted styrenes showed no product under the employed conditions. Inductive and mesomeric donor substituents generally gave excellent yields for hydroamination with lithium piperidide 2a. 4-Methylstyrene gave $90 \%$ of 3a. Yields of $73 \%$ for $4-O M e ~ 3 \mathbf{i}$ and $93 \%$ for $3-O M e ~ 3 \mathbf{j}$ are found. $3,4-$ Dimethoxystyrene $\mathbf{1 g}$ was hydroaminated to give $97 \%$ of $\mathbf{3 k}$. Rather electron-poor styrenes worked reasonably well, with 4vinylbiphenyl $1 \mathrm{~h}$ giving $59 \%$ of $3 \mathrm{p}$. The $3-\mathrm{CF}_{3}$ substituted $3 \mathrm{~m}$ was isolated in a $41 \%$ yield. The reactions with $4-F 1 j$ led to a mixture of products containing $3 \mathrm{n}$ in a $45 \%$ yield; whereas with $4-\mathrm{Cl} \mathbf{1 k}$, amine 30 was formed in a $64 \%$ yield.

Table 4. Reaction scope concerning various styrenes $\mathbf{1}$ with LiPip $\mathbf{2 a}$.

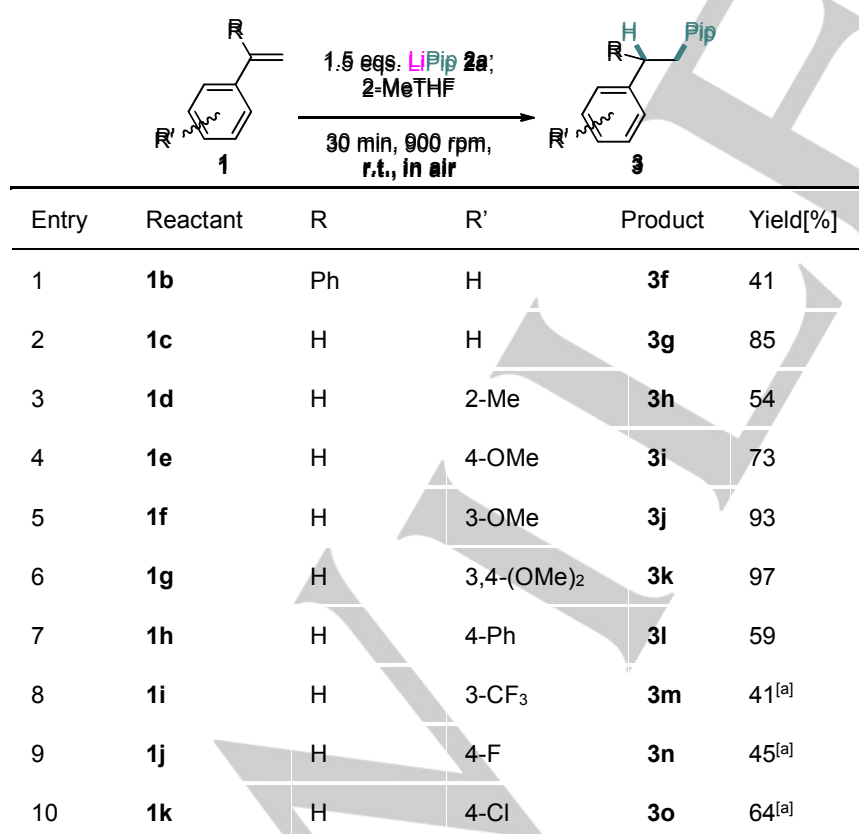

Reactions performed on a $1 \mathrm{mmol}$ scale in $1.5 \mathrm{~mL}$ of 2-MeTHF. They are stirred at $900 \mathrm{rpm}$ for $30 \mathrm{~min}$. Mean yields of isolated aliquots of at least two independent reactions are given. [a] Side reactions led to contaminated product. [

Finally, to probe the scope of this approach in terms of the nature of the group 1 metal amide we next studied the reactivity of $1 \mathrm{a}$ against NaPip (5a). While sodium amides are significantly more sensitive to air and moisture than their lithium counterparts, we found that using 2-MeTHF under air, the hydroamination product $\mathbf{3 a}$ is obtained in a $71 \%$ yield in just 5 min (Figure $2 \mathrm{i}$ ). Longer reaction times did not lead to higher conversions, suggesting that while the hydroamination process is faster than using 2 a (using LiPip after $5 \mathrm{~min} 3 \mathrm{a}$ is formed in a much lower $32 \%$ yield), the decomposition of the sodium intermediate in the presence of air and moisture also occurs more rapidly. Showing the beneficial and key role of moisture, if the reaction is carried out under inert atmosphere conditions and dry solvents polymerisation of styrene is observed.

(i)
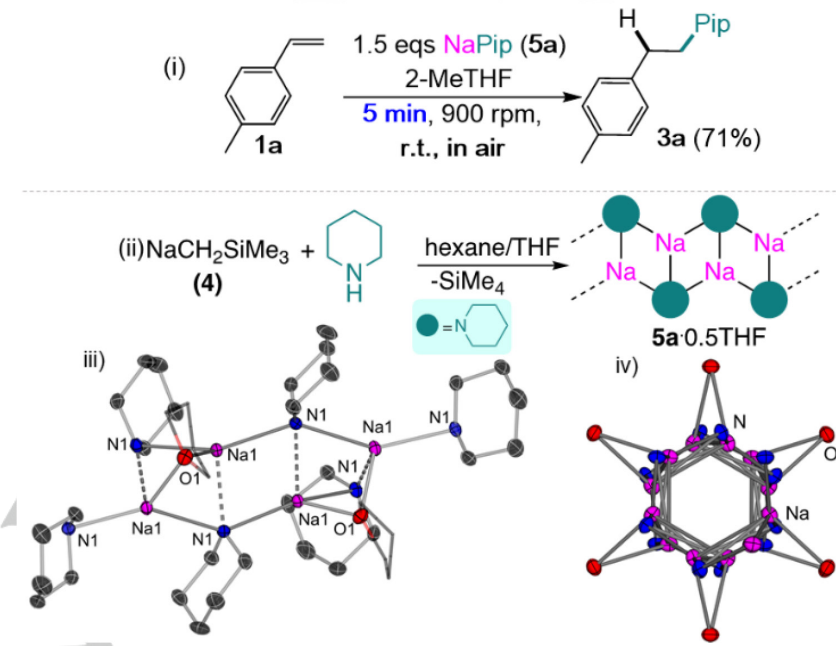

Figure 2. (i) Air and moisture compatible Na-mediated hydroamination of 1a in 2-MeTHF; (ii) Synthesis of 5a.0.5THF; (iii) portion of polymeric ladder structure of 5a.0.5THF; and (iv) axial view showing helical conformation of ladder framework (with thermal ellipsoids rendered at $50 \%$ probability, H's omitted for clarity and $\mathrm{C}$ atoms of THF molecules shown as wire frame for clarity; in (iv) C atoms have also been omitted).

To find out more about the constitution of NaPip, $\mathbf{5 a}$ (prepared by deprotonation of piperidine via $\mathrm{NaCH}_{2} \mathrm{SiMe}_{3}, 4$ ) was recrystalised in a mixture of hexane/THF affording $\left[\left\{\mathrm{Na}_{2} \mathrm{Pip}_{2}(\mathrm{THF})\right\}_{\infty}\right]$ (5a-0.5THF). Its structure was established by $X$-ray crystallography (Figure 2ii). Exhibiting a rare polymeric ladder structure, $5 \mathrm{a} \cdot 0.5 \mathrm{THF}$ is composed of four-membered $\left\{\mathrm{Na}_{2} \mathrm{~N}_{2}\right\}$ rings laterally associated, with a THF bridge connecting the two $\mathrm{Na}$ centres. Propagation occurs in a helical conformation with the THF molecules coordinated on the outer side of the helix (Figure 2 iii and iv). ${ }^{[17],[18]}$ Despite having this polymeric arrangement, 5 a is readily soluble in 2-MeTHF and $d_{8}$-THF, which suggests the formation of smaller aggregates. ${ }^{1} \mathrm{H}$ DOSY NMR experiments in $\mathrm{d}_{8}$-THF also suggest the formation of a monomer solvated by 3 molecules of $d_{8}$-THF $\left(M W_{\text {det }}=348 \mathrm{~g} / \mathrm{mol}\right.$, error $4 \%$, see SI for details), ${ }^{[19]}$ which is consistent with the fast reactivity seen when reacted with 1 a (Figure $2 \mathrm{i}$ ).

Inspired by these results we also pondered whether sodium alkyl 4 could act as a pre-catalyst for the hydroamination of styrenes under inert atmosphere conditions (to avoid competing decomposition pathways). While lithium alkyls have shown good promise in catalytic hydroamination of olefins, ${ }^{[3 c]}$ the use of sodium alkys has not been investigated. Interestingly, we found that using just $5 \mathrm{~mol} \%$ of $\mathbf{4}$ hydroamination of several styrenes [1a, 1c, and $\alpha$-Me-styrene (1I)] with a range of secondary amines [2a2d, $\left.\mathrm{HNBz}_{2}(\mathbf{2 f}), \mathrm{HNMeBz}(\mathbf{2 g}), \mathrm{HNBu}_{2}(\mathbf{2 h})\right]$ is accomplished in excellent yields $(72-88 \%)$ at room temperature in just 10 minutes 
(Scheme 4). The compatibility of the sodium alkyl 4 with THF is remarkable, just as its catalytic activity contrast with the sluggish reactivities reported for sodium catalysed hydroamination using $\mathrm{Na}$ metal or $\mathrm{NaH}$ as catalysts. ${ }^{[2 \mathrm{a}]}$

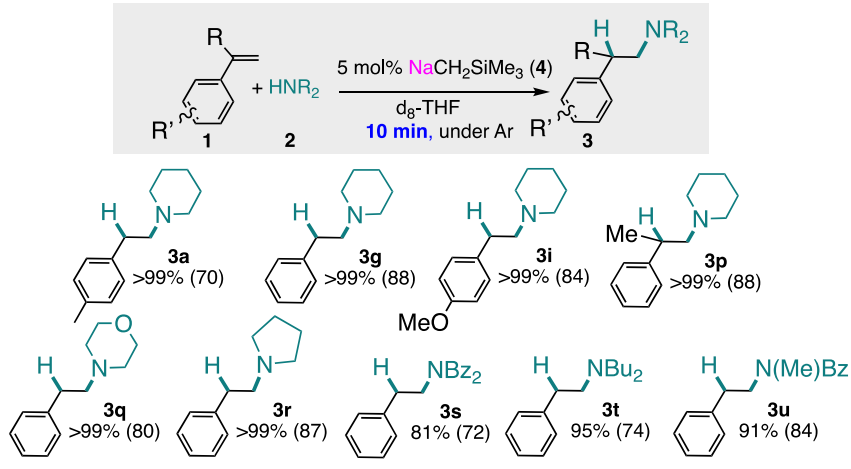

Scheme 4. Sodium catalysed hydroamination of vinyl arenes 1c, $1 \mathrm{~d}$ and $\mathbf{1 I}$ with amines 2a-d and 2f-2h. Conversions determined by NMR spectroscopy using ferrocene as internal standard (see SI for details); yields of isolated products shown in brackets.

To summarise, surprisingly we found that performing alkali-metalamide-executed intermolecular hydroamination reactions under air can be accelerated by moisture from ambient air, instead of being destroyed by its presence. This observation has led to the development of an exceptionally simple procedure for vinylarene hydroamination. Ambient moisture generates a steady supply of the necessary free amine to coordinate and so activate the alkalimetal amides then to quench the addition products. Formation of small kinetically activated aggregates of the metal amides seems to be key to enable hydroamination under these unconventional reaction conditions.

\section{Experimental Section}

Full experimental details and copies of NMR spectra are included in the Supporting Information.

\section{Acknowledgements}

We thank Robert Mulvey and Marina Uzelac for useful comments. Funding by the UK's Engineering and Physical Sciences Research Council (Grant number EP/S020837/1), ERC-Stg MixMetApps and the University of Bern is also acknowledged.

Keywords: s-block metals • hydroamination • solvent effects • air/moisture compatible $\bullet$ Lithium $\bullet$ catalysis

[1] (a) G. Osztrovszky, T. Holm, R. Madsen, Org. Biomol. Chem. 2010, 8 3402-3404; (b) M. J. Rodriguez-Alvarez, J. Garcia-Alvarez, M. Uzelac, M. Fairley, C. T. O'Hara, E. Hevia, Chem. Eur. J. 2018, 24, 1720-1725 (c) C. Vidal, J. Garcia-Alvarez, A. Hernan-Gomez, A. R. Kennedy, E. Hevia, Angew. Chem. Int. Ed. 2014, 53, 5969-5973; (d) C. Vidal, J. Garcia-Alvarez, A. Hernan-Gomez, A. R. Kennedy, E. Hevia, Angew. Chem Int Ed 2016, 55, 16145-16148; (e) A. Sánchez-Condado, G. A. Carriedo, A. Presa Soto, M. J. Rodríguez-Álvarez, J. García-Álvarez, E. Hevia, ChemSusChem 2019, 12, 3134-3143; (f) F. P. Perna, P. Vitale V. Capriati, Curr. Opin. Green Sustain. Chem. 2020, 21, 27-33; (g) L. Cicco, S. Sblendorio, R. Mansueto, F.M. Perna, A. Salomone, S. Florio, V. Capriati Chem. Sci. 2016, 7, 1192-1199.
[2] (a) T. E. Müller, K. C. Hultzsch, M. Yus, F. Foubelo, M. Tada, Chem. Rev. 2008, 108, 3795-3892; (b) J Seayad, A Tillack, C. G. Hartung, M. Beller, Adv. Synth. Catal. 2002, 344, 795-813; (c) M. C. Wood, D. C. Leitch, C S. Yeung, J. A. Kozak, L. L. Schafer, Angew. Chem. Int. Ed. 2007, 46, 354-358; (d) G. Tran, W. Shao, C. Mazet, J. Am. Chem. Soc. 2019, 141 14814-14822; (e) D. C. Miller, J. M. Ganley, A. J. Musacchio, T. C. Sherwood, W. R. Ewing, R. R. Knowles, J. Am. Chem. Soc. 2019, 141 16590-16594; (f) S. B. Herzon, J. F. Hartwig, J. Am. Chem. Soc. 2007 129, 6690-6691; (g) R. Kubiak, I. Prochnow, S. Doye, Angew. Chem. Int. Ed. 2009, 48, 1153-1156; (j) M. Beller, M. Eichberger, H. Trauthwein, Angew. Chem. Int. Ed. 1997, 36, 2225-2227.

[3] (a) K. Kumar, D. Michalik, I. Garcia Castro, A. Tillack, A. Zapf, M. Arlt, T. Heinrich, H. Böttcher, M. Beller, Chem. Eur. J. 2004, 10, 746-757; (b) P Horrillo-Martínez, K. C. Hultzsch, A. Gil, V. Branchadell, Eur. J. Org. Chem. 2007, 2007, 3311-3325; (c) S. Germain, M. Lecoq, E. Schulz, J. Hannedouche, ChemCatChem 2017, 9, 1749-1753; (d) R. J. Schlott, J. C. Falk, K. W. Narducy, J. Org. Chem. 1972, 37, 4243-4245; (e) J. Deschamp, J. Collin, J. Hannedouche, E. Schulz, Eur. J. Org. Chem. 2011, 2011, 3329-3338; (f) J. Hannedouche, E. Schulz, Chem. Eur. J. 2013, 19, 4972-4985.

[4] L. Davin, A. Hernán-Gómez, C. McLaughlin, A. R. Kennedy, R. McLellan, E. Hevia, Dalton Trans. 2019, 48, 8122-8130.

[5] (a) R. E. Mulvey, S. D. Robertson, Angew. Chem. Int. Ed. 2013, 52 11470-11487; (b) A. Pouilhès, J.-P. Baltaze, C. Kouklovsky, Synlett 2013, 24, 1805-1808

[6] J. Swarbrick, J. C. Boylan, Encyclopedia of Pharmaceutical Technology Dekker: New York 1988, pp 110-116.

[7] (a) S. Monticelli, L. Castoldi, I. Murgia, R. Senatore, E. Mazzeo, J.Wackerlig, E. Urban, T. Langer, V. Pace, Monatsh. Chem. 2017, 148 37-48; (b) V. Pace, P. Hoyos, L. Castoldi, P. Domínguez de María, A. R Alcántara, ChemSusChem 2012, 5, 1369-1379; (c) V. Pace, Aus. J. Chem. 2012, 65, 301-302; (d) D. F. Aycock, Org. Process Res. Dev. 2007, 11, 156-159.

[8] (a) P. Lei, Y. Ling, J. An, S. Nolan, M. Szostak, Adv. Synth. Catal. 2019 361, 5654-5660. (b) S. Monticelli, L. Castoldi, I. Murgia, R. Senatore, E. Mazzeo, J. Wackerlig, E. Urban, T. Langer, V. Pace, Monatsh. Chem. 2017, 148, 37-48; (c) D. F. Aycock, Org. Process Res. Dev. 2007, 11, $156-159$.

[9] M. Fairley, L. J. Bole, F. F. Mulks, L. Main, A. R. Kennedy, C. T. O'Hara J. García-Alvarez, E. Hevia, Chem. Sci., 2020, DOI: 10.1039/DOSC01349H.

[10] (a) V. Snieckus, M. Rogers-Evans, Lithium Piperidide. In: e-EROS Encyclopedia of Reagents for Organic Synthesis, John Wiley \& Sons, Ltd. Chichester 2001, pp 1-2; (b) N. Monck, P. Timm, Lithium Dicyclohexylamide. In: e-EROS Encyclopedia of Reagents for Organic Synthesis, John Wiley \& Sons, Ltd. Chichester 2005, pp 1-2.

[11] M. S. Hill, D. J. Liptrop, C. Wheetman, Chem. Soc. Rev. 2016, 45, 972.

[12] (a) D. W. Slocum, C. A. Jennings, J. Org. Chem. 1976, 41, 3653-3664 (b) A. G. M. Barrett, C. Brinkmann, M. R. Crimmin, M. S. Hill, P. Hunt, P. A. Procopiou, J. Am. Chem. Soc. 2009, 131, 12906-12907.

[13] (a) C. Brinkmann, A. G. M. Barrett, M. S. Hill, P. A. Procopiou, J. Am Chem.Soc. 2012, 134, 2193-2207; (b) M. De Tullio, A. Hernán-Gómez, Z. Livingstone, W. Clegg, A. R. Kennedy, R. W. Harrington, A. Antiñolo A. Martínez, F. Carrillo-Hermosilla, E. Hevia, Chem. Eur. J. 2016, 22 , $17646-17656$.

[14] X-ray crystallographic studies have already shown that LiPip can form coordination adduct with $\mathrm{PipH}$, see G. Boche, I. Langlotz, M. Marsch, K Harms, N. E. S. Nudelman, Angew. Chem. Int. Ed. 1992, 31, 1205-1207.

[15] G. Ndebeka, P. Caubère, S. Raynal, S. Lécolier, Polymer 1981, 22, 347 355; (b) G. Ndebeka, P. Caubère, $S$. Raynal, S. Lécolier, Polymer 1981 22, 356-360; (c) S. Carlotti, P. Desbois, V. Warzelhan, A. Deffieux, Polymer 2009, 50, 3057-3067; (d) A. C. Angood, S. A. Hurley, P. J. Tait, J. Polym. Sci. Pol. Chem. 1973, 11, 2777-2791.

[16] In recent studies comparing the constitution of lithium anilides in THF and Me-THF we have shown that in all cases the same level of aggregation and solvation is observed in both solvents (see reference [9]) Considering also the greater solubility of lithium amides in 2-MeTHF in comparison with THF, formation of a similar $\left[\mathrm{Li}(\mathrm{Pip})(2-\mathrm{Me}-\mathrm{THF})_{3}\right]$ monomer can be proposed.

[17] For other examples of $\mathrm{Na}$ amide ladder structures see: (a) W. Clegg, K Henderson, L. Horsburgh, F. M. Mackenzie, R. E. Mulvey. Chem. Eur. J. 1998, 4, 53 - 56; (b) N. Kuhn, G. Henkel, J. Kreutzberg, Angew. Chem Int. Ed. 1990, 29, 1143-1144; (c) D. R. Baker, R. E. Mulvey, W. Clegg, P. A. O'Neil, J. Am. Chem. Soc. 1993, 115, 6472-6473.

[18] This structure complies with the ring-laddering principle, see (a) D. R. Armstrong, D. Barr, W. Clegg, R. E. Mulvey, D. Reed, R. Snaith, K. Wade, Chem. Commun., 1986, 869; (b) R. E. Mulvey, Chem. Soc. Rev., 1991 20, 167 (c) R. E. Mulvey, Chem. Soc. Rev., 1998, 27, 339. 
Entry for the Table of Contents (Please choose one layout)

\section{COMMUNICATION}

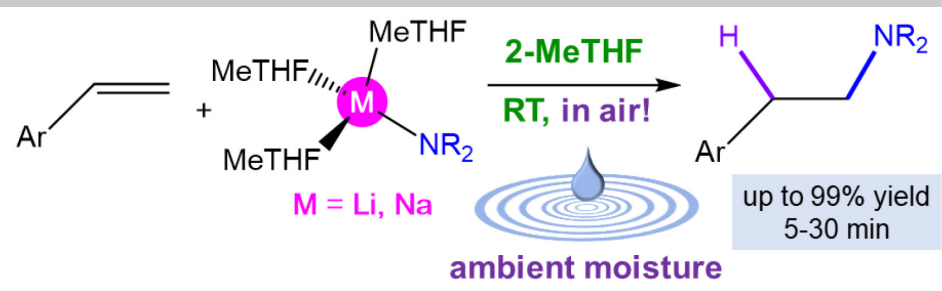

Moisturising for Smoother Reactions: The nemesis of organo-alkali metal reagents, ambient moisture can remarkably accelerate the alkali-metal amide induced hydroamination of styrenes. A practically simple aerobic procedure for these hydroaminations under air and in renewable 2-methyltetrahydrofuran is revealed.
F. F. Mulks, L. J. Bole, L. Davin, A. Hernán-Gómez, A. R. Kennedy, J. García-Álvarez* and E. Hevia*

Page No. - Page No.

Ambient Moisture Accelerates Hydroamination Reactions of Vinylarenes with Alkali-Metal Amides under Air 